\title{
APORTE Y CARACTERÍSTICAS DEL CUENTA PROPIA EN EL MERCADO LABORAL HONDUREÑO
}

\author{
ISSN 2219-6722 \\ ISSNE 2222-2707 \\ KENIA MEZA, Instituto de Investigaciones Económicas y Sociales \\ Universidad Nacional Autónoma de Honduras \\ kmeza@iies-unah.org \\ JOSÉ BAYARDO CABRERA, Instituto de Investigaciones Económicas y Sociales \\ Universidad Nacional Autónoma de Honduras \\ jcabrera@iies-unah.org \\ CINTHYA ARTEAGA, Instituto de Investigaciones Económicas y Sociales \\ Universidad Nacional Autónoma de Honduras \\ carteaga@iies-unah.org
}

\section{RESUMEN}

La necesidad de identificar los segmentos en los cuales el mercado laboral de Honduras está estructurado representa un reto en materia de inicialmente identificar la caracterización de los mismos, en ese sentido la presente investigación muestra una detallada descripción del trabajo por cuenta propia de Honduras para el periodo 2013 al 2015; definiendo a estos como aquellas personas que trabajan de manera independiente, usualmente con baja productividad y con salarios bajos, usualmente de baja calificación profesional.

En el marco sobre la teoría de la heterogeneidad del trabajo por cuenta propia y de cuál es la dinámica de este sector en Honduras, desarrollamos una serie de análisis frente al nivel educativo, el salario, rama de actividad económica entre otras variables que nos permitirán presentar de manera descriptiva este sector poco explorado aún en nuestro país, pero que merece especial atención, considerando los elementos de transición demográfica y las políticas económicas actuales, que en cierto sentido podrían incentivar el aumento de este segmento del mercado laboral.

Palabras claves: Mercado laboral, Sector informal, Cuenta Propias, Emprendimiento. 


\title{
CONTRIBUTION AND CHARACTERISTICS OF SELF-EMPLOYED IN THE LABOR MARKET HONDUREÑO
}

ISSN 2219-6722

ISSNE 2222-2707

KENIA MEZA, Instituto de Investigaciones Económicas y Sociales Universidad Nacional Autónoma de Honduras

kmeza@iies-unah.org JOSÉ BAYARDO CABRERA, Instituto de Investigaciones Económicas y Sociales Universidad Nacional Autónoma de Honduras jcabrera@iies-unah.org

CINTHYA ARTEAGA, Instituto de Investigaciones Económicas y Sociales Universidad Nacional Autónoma de Honduras carteaga@iies-unah.org

\begin{abstract}
The need to identify segments in which the labor market in Honduras is structured represents a challenge in terms of initially identifying the characterization of the same, in that sense this research shows a detailed description of self-employment of Honduras for the period 2013 to 2015; defining them as those who work independently, often with low productivity and low wages, usually low professional qualification.

In the framework of the theory of the heterogeneity of self-employment and what is the dynamics of this sector in Honduras, we developed a series of tests versus education level, salary, branch of economic activity and other variables that will allow us to resent descriptively this sector still little explored in our country, but it deserves special attention, considering the elements of demographic transition and the current economic policies, which in a sense could encourage the increase of this segment of the labor market.
\end{abstract}

Key words: Labor Market, Informal Sector, Self-employment, Entrepreneurship. 


\section{INTRODUCCIÓN}

En la economía se ha generado como un mundo paralelo al sector formal que se puede conocer como economía paralela, mercado subterráneo, mercado negro o simplemente sector Informal que se entiende como el conjunto de unidades que producen bienes y servicios a pequeñas escalas con herramientas rudimentarias, destacando que es generador de empleo (ocasionales, familiares, sociales, entre otras) y más significativamente a la población pobre (Organización Internacional del Trabajo [OIT], 2013).

Entendiéndose como empleo informal todos aquellos que no se encuentran sometidos a la legislación nacional, no tiene protección social ni beneficios laborales, cubriendo todas las situaciones de empleo: empleadores, asalariados, trabajadores por cuenta propia, trabajadores auxiliares (OIT, 2013). Aunque esta definición dependerá de cada país en el caso de Costa Rica define empleo informal como personas sin seguridad social, que reciben pagos en especie o una sola vez, personas sin remuneraciones, la cuenta propia que no tienen constituida su sociedad y los ocasionales que laboran por un mes, debido a ello no están inscritas y no cuentan contabilidad (Instituto Nacional de Estadística y Censos [INEC], 2014).

Debido a la importancia que tiene el empleo informal particularmente en Latinoamérica que existen 130 millones que representan un 47\%, de los cuales $30.5 \%$ del empleo informal es del sector informal no agrícola que corresponden a empleadores y cuenta propias; un $11 \%$ del empleo informal en el sector formal y un $4.9 \%$ del sector de los hogares (Servicios Domésticos) (OIT, 2014).En el mismo informe se destaca que el mayor porcentaje de informalidad es en trabajadores por cuenta propia $(82.3 \%)$ ya que no cuenta con empresas constituidas, trabajadores domésticos un (77.4\%) y los microempresarios máximos de 10 empleados (58.6\%); representando 79\% del empleo informal. Destacando que el empleo en las microempresas es 4 veces más que las empresas de más de 10 empleados.

Debido que el sector informal está compuesto considerablemente en pequeñas empresas o empresas de cuenta propia; que no cuentan con organización y capital, sin embargo, sin son generadoras de empleo informal. Entendiendo empresa como toda unidad que produce bienes y servicios, y no solamente las que emplean personal sino a también a las que operan como cuenta propia o con ayuda familiar (OIT, 2013).

En el caso de Honduras se ha caracterizado por la informalidad en el mercado laboral, no obstante, no toda la población que labora en dicho mercado tiene un nivel educativo bajo mostrando que el emprendedurismo ha llegado ser parte de la informalidad debido a que no acceden a la seguridad social o no se constituyen como empresa, aunque presten un servicio. Aunado a ello esta investigación está orientada en realizar una descripción del mercado laboral de Honduras enfocado en el autoempleo deter- 
minando qué porcentaje representan, el nivel educativo alcanzado, conocer la edad del cuentapropista, indagar su dominio, en que rama de actividad se localizan y establecer qué porcentajes de ellos cuentan con seguridad social. La investigación muestra en el capítulo I, los antecedentes de las investigaciones relacionadas con el cuentapropista, un establecimiento teórico y un marco conceptual y el capítulo II, se realiza una caracterización de los cuentapropistas en el periodo 2013-2015.

\subsection{MARCO TEÓRICO}

\subsubsection{Antecedentes}

En el Informe de Desarrollo Mundial, se menciona ¿Que si es posible fomentar el espíritu emprendedor?, sin embargo, en los países en desarrollo las empresas y microempresas que se crean son de supervivencia; mirándose menguadas las posibilidades de crecimiento y siendo en su mayoría cuentapropistas, aunque generen empleos el apoyo financiero es casi nulo para dichas empresas (Banco Mundial, 2013). Así también el banco realiza una comparación entre regiones, destacando que, aunque se generen puestos de trabajo no necesariamente son remunerados como el caso de África al sur del Sahara que más del $80 \%$ de los empleos son sin remuneraciones ya sea por cuenta propia o trabajo agrícola, caso contrario el de Europa y Asia Central que más un de un $75 \%$ son empleos asalariados (vea gráfico $\mathrm{N}^{\circ} 1$ ).

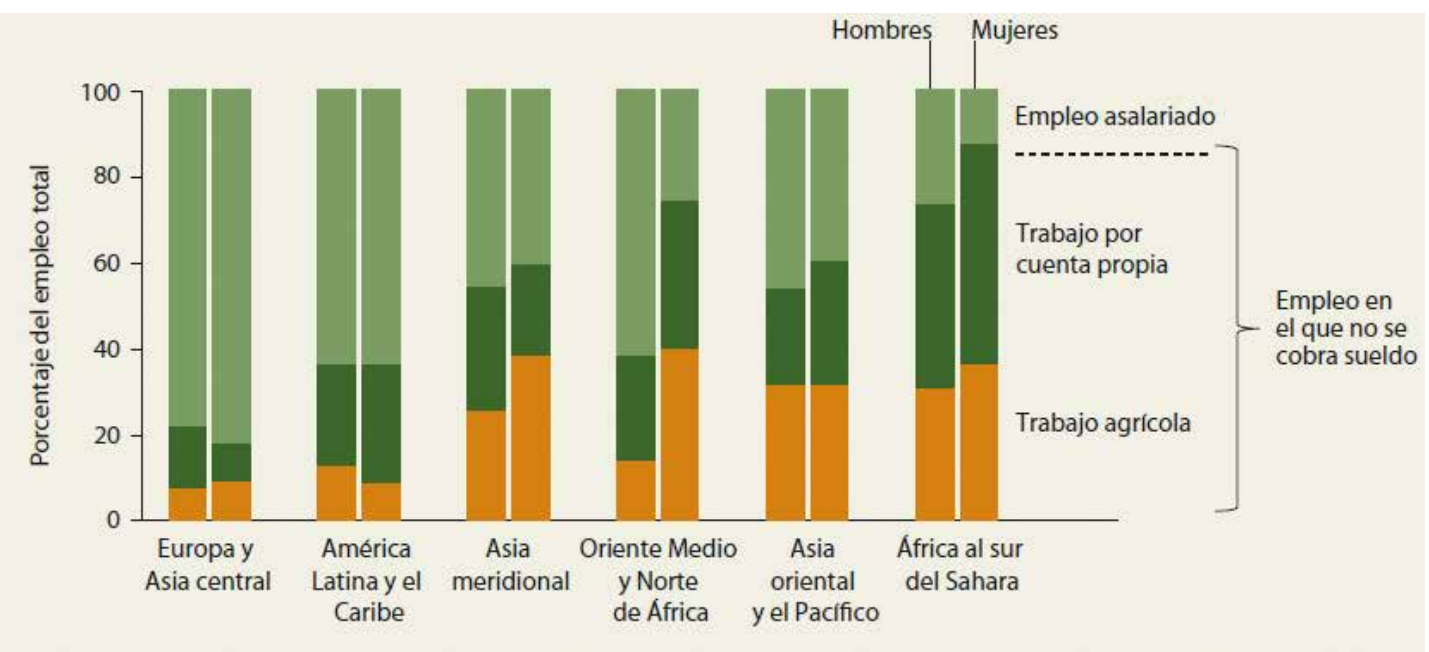

Figura No. 1. El empleo no siempre va acompañado de un sueldo Fuente: Informe de sobre el Desarrollo Mundial 2013

Nota: Los valores corresponden al año más reciente para el que se dispone de datos 
Se ha definido como cuenta propia o autónoma a una persona que trabaja para sí misma, que carecen de un contrato de unos empleados ya que reciben el ingreso directamente de una persona o empresa con el distintivo que se conoce sus ingresos. Los independientes no tienen seguridad social, ni declaran sus ingresos. Existiendo dos tipos de cuentapropista formales lo que llevan registros de sus ingresos y los que declaran; pagando impuestos y sus cotizaciones y siendo caso contrario a los informales (Asociación Internacional de Seguridad Social [ISSA], 2012)

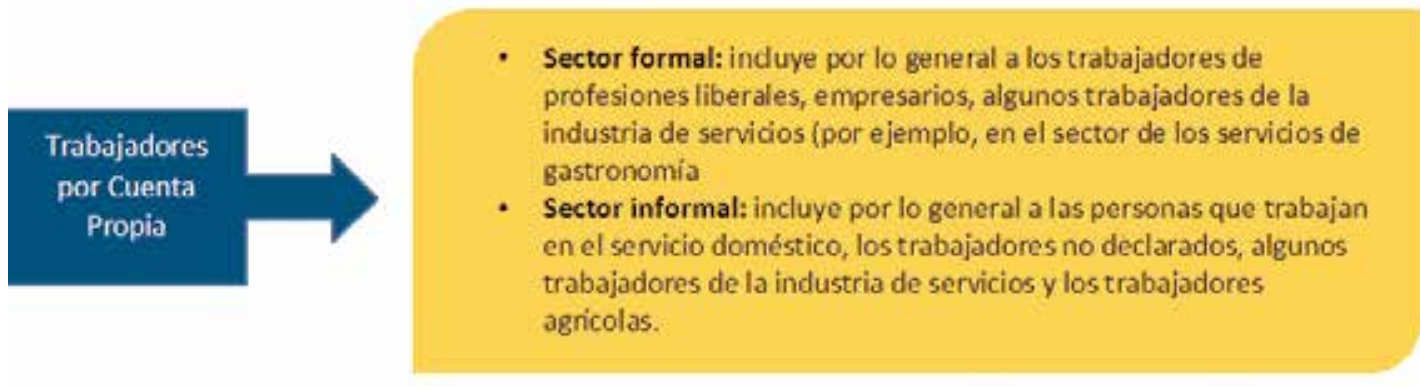

\section{Figura No. 2. Definición de Cuenta propia \\ Fuente: Figura tomada del Manual sobre la extensión de la cobertura de seguridad social a los trabajadores por cuenta propia.}

Amarante y Perazzo (2013) realizaron una investigación para Uruguay, mencionando sobre la medida que se aplicó al pago de un monotributo de las empresas que realizan una producción mínima a la economía; realizando un único pago de impuesto y así como el aporte a la seguridad social. Su investigación se orienta a la cuenta propia que poseen local y a los que no, mostrando que los autónomos sin local son más afectados por los comportamientos de la economía. Asimismo, que los sin local se ubican más en los sectores de comercio y construcción (siendo más susceptible a los ciclos económicos) y el caso de los que tienen local el sector industria y comercio. Determinando que la permanencia en el puesto de trabajo de los sin local un $25.13 \%$ tenía menos de un año y entre 11-10 años los con local.

Existen distintas razones para que el individuo se incentive para ser cuenta propia ya sea el emprendedurismo o encontrarse en desempleo. Donde el individuo puede estar en distintos segmentos laborales: trabajador por cuenta propia, empleada, asalariada, desempleada e inactiva. Entre las características del trabajador independiente se destacan baja escolaridad (lo que provoca que individuo al momento de trasladarse en otro segmento lo haga a desempleado), mayormente son hombres, poco nivel de riesgo, tipo de establecimiento rudimentarios y edad son personas mayores (Cea, Contreras, Martínez \& Puentes, 2009).

En el caso de Argentina donde Lepore \& Schleser (s.f), describen a la cuenta propia 
como muy heterogéneos, ya que se pueden encontrar médicos, abogados, contadores; así como comerciantes, albañiles, vendedores ambulantes, etc. Definen como cuenta propia como los que trabajan de manera independiente o con un socio, pero no contratan de forma periódica empleados y la cuenta propia típicos que trabajan según lo impuesto por sus clientes o empleadores. Teniendo como categorías de: patrón o empleador, trabajador por cuenta propia, obrero o empleado y trabajador sin salario. Establecen tres categorías laborales para los cuentapropistas las cuales se visualizan en la Figura No. 2.
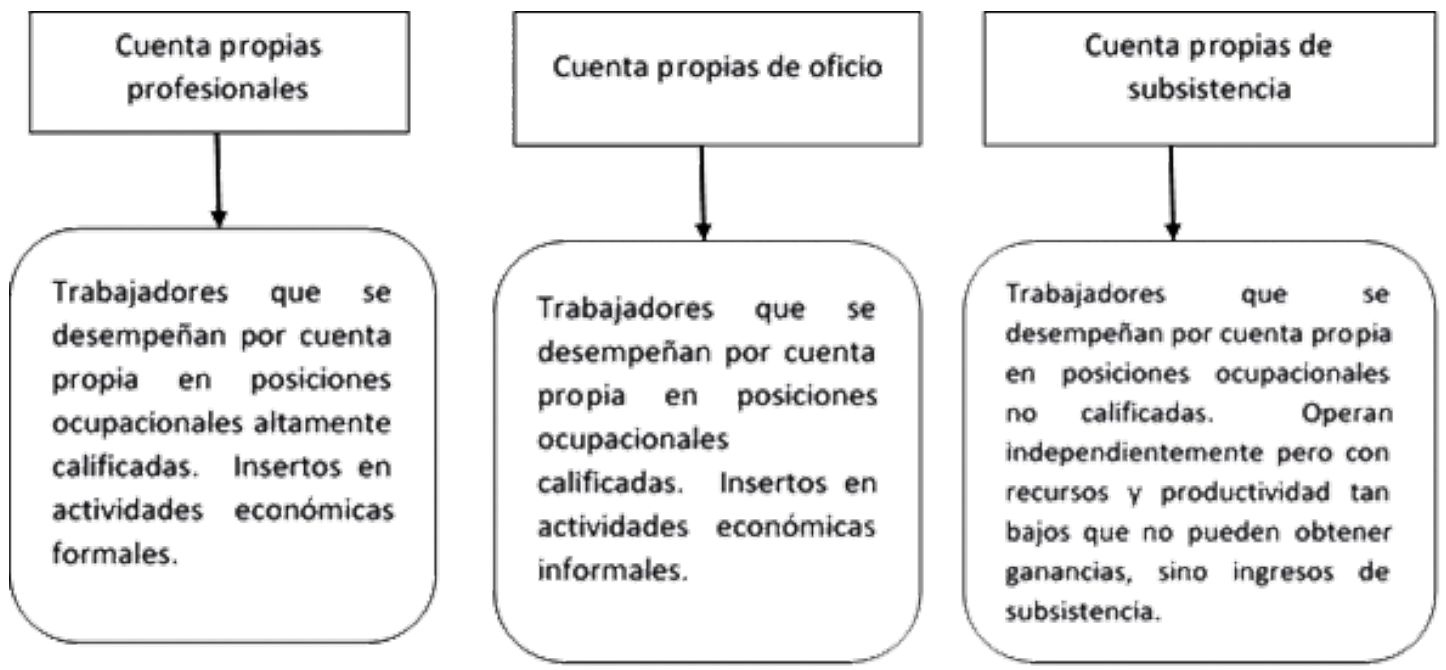

\section{Figura No. 3. Clasificación de la Cuenta Propia Fuente: elaboración propia con datos de Lepero y Scheler (2004).}

Se destaca que la investigación que los empleados profesionales tienen mejores ingresos, menos horas laborales, caso contrario a lo de subsistencia que fueron salarios inferiores al igual que los técnicos, la cuenta propia por oficio en el 2004 en Argentina representaban $78 \%$, por subsistencia un $12 \%$ y los profesionales un $10 \%$. En caso de las mujeres fueron más representativas como profesionales participando como cuenta propia y el hombre en la categoría por subsistencia y en cuanto la edad de 30-59 años mostró estar ubicados en autoempleo.

\subsubsection{Teorías}

En el análisis del mercado de trabajo entra en la discusión la formalidad y la informalidad en la que se desarrollan los ocupados de la Población Económicamente Activa (PEA) del país, en ese sentido la Organización Internacional del Trabajo (OIT, 2013) plantea que la importancia del mercado informal se debe a que es la principal fuente de oportunidades de empleo en muchos países y que la composición del sector informal de 
la economía se compone de empresas pequeñas o de empresas por cuenta propia y con muy bajos niveles de organización formal o capital.

En el análisis de la estructura o composición de los ocupados en los diferentes mercados de trabajo, es necesario que se analice los diferentes niveles de ocupación su caracterización y comportamiento con el objetivo de identificar los elementos que pueden ser incidentes en el desarrollo económico de un país. Lepore \& Schleser (2004) definen que existe una relación inversa entre el nivel de desarrollo económico y la participación del cuentapropismo en la estructura ocupacional, en este sentido podría considerarse que los efectos del desempleo pueden ser una de las causas que impulsan el trabajo por cuenta propia o autoempleo.

Existe un consenso sobre la incidencia de la heterogeneidad estructural de los mercados de trabajo de América Latina y cómo este incide en el comportamiento de los ocupados por cuenta propia o autoempleo. Según Lepero y Scheler (2004) la hipótesis inicial sobre este análisis es que "las economías subdesarrolladas se caracterizan por una conformación dual de sus estructuras productivas, en la que coexiste un sector atrasado o informal junto con un sector moderno o formal, de avanzada tecnología y elevada productividad". En este sentido la consideración de la productividad como medida de desarrollo de un país también incide en la evolución directa de la estructura del mercado laboral.

Según la teoría Schumpeteriana un emprendedor se define como "el fundador de una nueva empresa, un innovador que rompe con la forma tradicional de hacer las cosas, con las rutinas establecidas. Debe ser una persona con dotes de liderazgo, y con un talento especial para identificar el mejor modo de actuar" (Schumpeter, 1934). También se expone que el emprendedor tiene la habilidad de ver las cosas como nadie las ve.

En la teoría del Desarrollo Económico de Schumpeter identifica tres clases de elementos que afectan al emprendedor: i.-la introducción de un nuevo bien, servicio, proceso de producción, apertura de un nuevo mercado, entre otros relacionados con la producción, distribución y consumo. ii.-Las formas de motivación empresarial y iii.-los factores que inhiben la actividad emprendedora (Carrasco y Castaño, 2008).

\subsubsection{Marco Conceptual}

Bajo la teoría de la heterogeneidad estructural de los mercados de trabajo según Lepero y Scheler (2004) el sector informal lo define como "un submodo de producción específica que reúne a las unidades con escasa capacidad de acumulación", los autores también destacan que una de las características del mercado informal es "la facilidad de acceso o entrada determinada por los bajos niveles de calificación e inversión requeridos para su desempeño".

En cuanto a los cuentapropistas se podría definir que están caracterizados por la producción mercantil simple, con mínimo de capital de entrada les proporciona ingresos similares a los de sus pares asalariados en los mismos sectores de actividad económica. Se trata de un sector de los ocupados en el que predomina la lógica de la supervivencia 
como estrategia de inserción socio-laboral.

También los cuentapropistas son considerados "agentes adversos al riesgo", asociados a personas con cierta disposición a asumir riesgos en algún grado de iniciativa y capacidades de gestión de negocios a micro escala (Lepero y Scheler, 2004), definiéndolos, así como emprendedores.

\section{Tabla No. 1 .Caracterización de metodologías para la estimación de la informalidad}

\begin{tabular}{|c|c|c|c|}
\hline Características & $\begin{array}{l}\text { Programa } \\
\text { Regional del } \\
\text { Empleo para } \\
\text { América Latina } \\
\text { y el Caribe } \\
\text { (PREALC- } \\
\text { OIT) }\end{array}$ & $\begin{array}{lr}\text { XV } & \text { Conferencia } \\
\text { Internacional } & \text { de } \\
\text { Estadísticos del Trabajo } \\
\text { (CIET) (sector informal) }\end{array}$ & $\begin{array}{l}\text { XVII Conferencia Internacional } \\
\text { de Estadísticos del Trabajo } \\
\text { (CIET) (empleo informal) }\end{array}$ \\
\hline Zona y rama & $\begin{array}{l}\text { Urbano }- \text { No } \\
\text { agrícola. }\end{array}$ & $\begin{array}{l}\text { Debería abarcar zonas } \\
\text { urbanas y rurales. Por } \\
\text { razones prácticas, puede } \\
\text { limitarse el ámbito del } \\
\text { sector informal a las } \\
\text { empresas de los hogares } \\
\text { que realizan actividades no } \\
\text { agrícolas. }\end{array}$ & $\begin{array}{l}\text { Debería abarcar zonas urbanas } \\
\text { y rurales. Los países que } \\
\text { excluyen actividades agrícolas } \\
\text { del ámbito de sus estadísticas } \\
\text { sobre el sector informal, } \\
\text { deberían elaborar definiciones } \\
\text { adecuadas de los tipos de } \\
\text { empleo informal no asalariado } \\
\text { en la agricultura }\end{array}$ \\
\hline $\begin{array}{l}\text { Situación en el } \\
\text { empleo }\end{array}$ & $\begin{array}{l}\text { Trabajadores } \\
\text { independientes } \\
\text { o por cuenta } \\
\text { propia (excluye } \\
\text { profesionales y } \\
\text { técnicos). }\end{array}$ & $\begin{array}{l}\text { Empresas informales de } \\
\text { trabajadores por cuenta } \\
\text { propia (incluyendo } \\
\text { profesionales y técnicos si } \\
\text { no tienen condiciones de } \\
\text { formalidad, en especial } \\
\text { registro conforme a la } \\
\text { legislación nacional). } \\
\text { Pueden incluirse todas las } \\
\text { empresas por cuenta propia } \\
\text { o solamente las que no } \\
\text { están registradas. }\end{array}$ & $\begin{array}{l}\text { Trabajadores por cuenta propia } \\
\text { dueños de sus propias empresas } \\
\text { del sector informal (incluyendo } \\
\text { profesionales y técnicos si no } \\
\text { tienen condiciones de } \\
\text { formalidad, en especial registro } \\
\text { conforme a la legislación } \\
\text { nacional). Pueden incluirse } \\
\text { todos los trabajadores por } \\
\text { cuenta propia dueños de sus } \\
\text { propias empresas informales o } \\
\text { solamente los que no están } \\
\text { registrados }\end{array}$ \\
\hline
\end{tabular}




\begin{tabular}{|c|c|c|c|}
\hline & $\begin{array}{l}\text { Empleadores o } \\
\text { propietarios de } \\
\text { empresas de } \\
\text { cinco o menos } \\
\text { ocupados. }\end{array}$ & $\begin{array}{l}\text { Empresas de empleadores } \\
\text { informales en función de } \\
\text { una o varias condiciones: } \\
\text { (i) el tamaño de las } \\
\text { unidades es inferior a un } \\
\text { nivel determinado de } \\
\text { empleo; y (ii) no están } \\
\text { registradas o no ocupan } \\
\text { empleados registrados. }\end{array}$ & $\begin{array}{l}\text { Empleadores dueños de sus } \\
\text { propias empresas del sector } \\
\text { informal en función de una o } \\
\text { varias condiciones: (i) el } \\
\text { tamaño de las unidades es } \\
\text { inferior a un nivel determinado } \\
\text { de empleo; y (ii) no están } \\
\text { registradas o no ocupan } \\
\text { empleados registrados }\end{array}$ \\
\hline & $\begin{array}{l}\text { Asalariados en } \\
\text { empresas de } \\
\text { cinco o menos } \\
\text { ocupados. }\end{array}$ & $\begin{array}{l}\text { Asalariados ocupados en } \\
\text { empresas informales en } \\
\text { función de una o varias } \\
\text { condiciones: (i) el tamaño } \\
\text { de las unidades es inferior a } \\
\text { un nivel determinado de } \\
\text { empleo; y (ii) no están } \\
\text { registradas o no ocupan } \\
\text { empleados registrados. }\end{array}$ & $\begin{array}{l}\text { Asalariados que tienen empleos } \\
\text { informales, tanto si están } \\
\text { empleados por empresas del } \\
\text { sector formal o por empresas } \\
\text { del sector informal }\end{array}$ \\
\hline & $\begin{array}{l}\text { Trabajadores } \\
\text { familiares o } \\
\text { auxiliares no } \\
\text { remunerados }\end{array}$ & $\begin{array}{l}\text { Trabajadores familiares o } \\
\text { auxiliares ocupados en } \\
\text { empresas de empleadores } \\
\text { informales o en empresas } \\
\text { de trabajadores por cuenta } \\
\text { propia informales. }\end{array}$ & $\begin{array}{l}\text { Trabajadores familiares o } \\
\text { auxiliares, independientemente } \\
\text { de si trabajan en empresas del } \\
\text { sector formal o informal }\end{array}$ \\
\hline & & $\begin{array}{l}\text { Miembros de cooperativas } \\
\text { de productores que no están } \\
\text { formalmente constituidas } \\
\text { como entidades legales. }\end{array}$ & $\begin{array}{l}\text { Miembros de cooperativas de } \\
\text { productores que no están } \\
\text { formalmente constituidas como } \\
\text { entidades legales. }\end{array}$ \\
\hline & $\begin{array}{l}\text { Servicio } \\
\text { doméstico }\end{array}$ & $\begin{array}{l}\text { El servicio doméstico se } \\
\text { excluye del ámbito del } \\
\text { sector informal, } \\
\text { identificándose de manera } \\
\text { separada. }\end{array}$ & $\begin{array}{l}\text { Asalariados de los hogares que } \\
\text { tienen empleos informales }\end{array}$ \\
\hline & & & $\begin{array}{l}\text { Trabajadores en actividades no } \\
\text { especializadas de producción o } \\
\text { bienes para consumo final de su } \\
\text { propio hogar }\end{array}$ \\
\hline $\begin{array}{l}\text { Unidad de } \\
\text { observación }\end{array}$ & $\begin{array}{l}\text { Las personas } \\
\text { ocupadas. }\end{array}$ & $\begin{array}{l}\text { Las unidades } \\
\text { producción. }\end{array}$ & Los Empleos \\
\hline
\end{tabular}

Fuente: Tomada de OIT, (información del PREALC y de la XV y XVII CIET) 


\section{METODOLOGÍA}

Con el propósito de identificar y desarrollar un análisis orientad a la búsqueda de la información para la caracterización del segmento del mercado laboral, la presente investigación se desarrolló con un enfoque cuantitativo, cuyo alcance será descriptivo y con un diseño no experimental de corte longitudinal para el periodo 2001-2015 con el propósito de analizar la variable cuenta propia o autoempleo, y utilizaremos un diseño no experimental de corte transversal para la caracterización de las personas que se catalogan como parte de los trabajadores de cuenta propia y autoempleo del periodo de análisis del 2013 al 2015. Ambos análisis con datos de la Encuesta Permanente de Hogares de Propósitos de Múltiples (EPHPM) para el mismo periodo de análisis, el cual es elaborado por Instituto Nacional de Estadísticas (INE).

\section{ANÁLISIS DE RESULTADO}

En este capítulo se hace una breve descripción del mercado laboral y una caracterización del cuentapropista hondureño. Resaltando su importancia como generador de empleo, su tendencia y analizando su comportamiento en el periodo 2013-2015. El mercado laboral hondureño se caracteriza por su alta informalidad, así también por las anomalías del mercado generando subempleados (visible e invisible) provocando una subestimación de la Tasa de Desempleo (TDA). Para el año 2015 según el INE la proporción de fuerza laboral que la economía no utilizó fue de $7.3 \%$ superior en $2 \mathrm{pp}$ al 2014, la Tasa Ocupacional (TO) que nos indica la demanda laboral a nivel nacional fue de $54.01 \%$ superior en $0.98 \mathrm{pp}$ al 2014 y el grado de participación nacional de $58.3 \%$ medido a través de Tasa de Participación (TP) en área urbana de 57.1\% y rural de 59.8\%.

El INE define los ocupados como todas las personas de 10 años y más que en la semana anterior a la realización de la Encuesta Permanente de Hogares de Propósitos Múltiples (EPHPM) trabajaron una hora, por lo menos, en un empleo, negocio propio o como familiares no remunerados. Honduras define "Trabajador Autónomo por cuenta Propia", como aquellas personas que ejercen de forma subordinada, con sus propios medios y capital, la autonomía y sin trabajadores asalariados a su cargo, realizando actividades productivas en su forma individual o colectiva con fines comerciales que constituyen su principal fuente de ingreso, (Artículo 3, ley de protección laboral para los trabajadores autónomos por cuenta propia y no asalariados.

La categoría ocupacional que se entiende como la relación de producción ${ }^{1}$ observándose en figura No. 3 según la actividad principal en el periodo 2001-2015 mostrando a los cuentapropistas con una proporción significativa en la población ocupada, no

1 Relaciones de Producción: de acuerdo a la posición o cargo que un trabajador tenga en su empleo puede ser empleador, empleado o ser trabajador por cuenta propia.

176 Revista Economía y Administración (E\&A) / V0L. 6 
obstante, los asalariados son los que han tenido una mayor proporción esto debido a que se componen por empleados públicos, privados y domésticos. Para el año 2015 de cada 100 empleados 47 correspondió personas con un patrono entendiéndose como las personas asalariadas (6 sector público, 38 empleado público y 3 al empleo doméstico), de cada 100 ocupados 40 corresponde a empleos autónomos y 13 a los empleados sin remuneración salarial. Contribuyendo a identificar la importancia del autoempleo en el mercado laboral hondureño en el 2015 fueron superior en $2.84 \mathrm{pp}$ a los empleados privados (siendo $40.26 \%$ cuenta propia y $37.41 \%$ los empleados privados) y para el 2014 un 2.07 pp (cuenta propia $40.69 \%$ y privados $38.62 \%$ ). Dejando en evidencia el mercado laboral del país debido a que no se identifica directamente si es por emprendedurismo, subsistencia o por oficio que la población opera como autónomos y en cierta medida agudizando los problemas laborales existen.

\section{Figura No. 4. Categorización de Ocupados por Actividad Principal periodo 2001-2015}

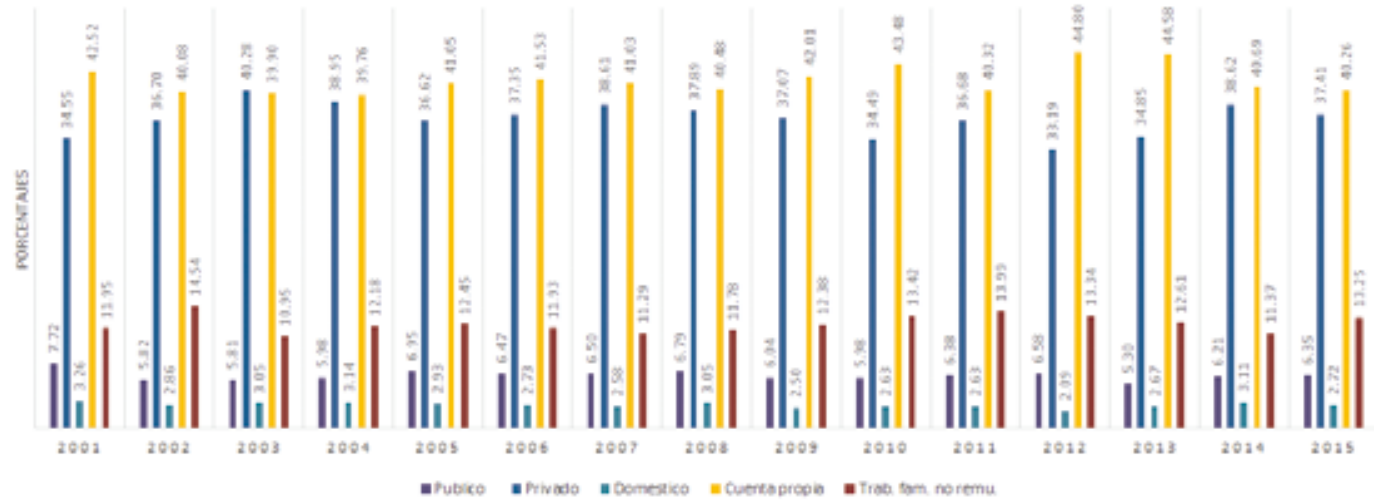

Fuente: Elaboración propia con datos del Instituto Nacional de Estadísticas (INE)

Se menciona reiteradamente la importancia de la mujer en el mercado laboral y su efecto en la reducción de la pobreza. Para el periodo de estudio 2013-2015 según la EPHPM la mujer ha incursionado como cuentapropista en promedio un $40 \%$ lo cual nos indica que su tendencia ha sido casi similar en los tres años. En el 2015 según empleo por género las mujeres ocupadas en el sector privado eran $27.3 \%$, público un $53.2 \%$, domésticos un $92 \%$, trabajos sin remuneración salarial un $41.3 \%$ y cuenta propia un $41.26 \%$. Dando a conocer que los empleos domésticos y públicos son únicos que la mujer tiene una mayor proporción de participación siendo los empleos con menos población laborando los públicos y los empleos domésticos con bajos salarios mostrando la vulnerabilidad de la mujer en el mercado laboral en Honduras (Ver figura No. 4). 


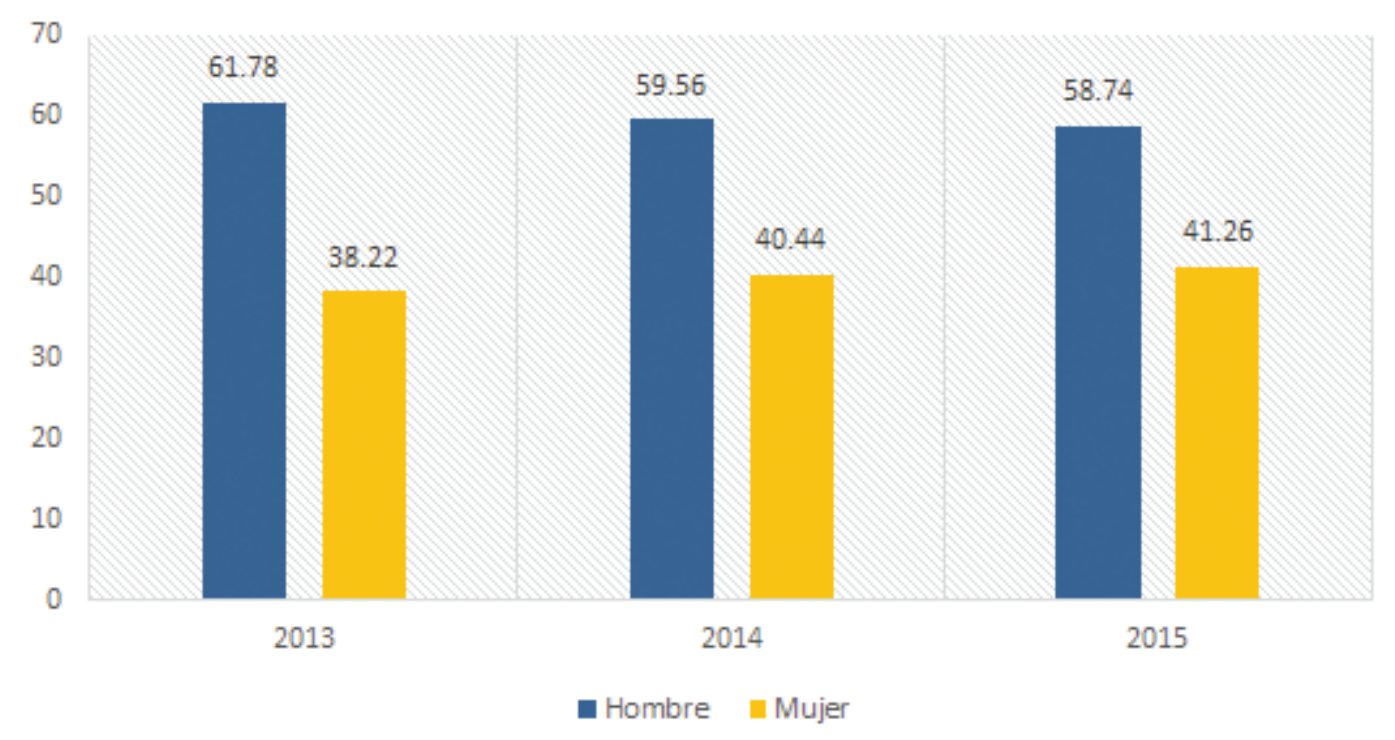

Figura No. 5. Porcentaje de Cuenta Propias por Género periodo 2013-2015 Fuente: Elaboración propia con datos del Instituto Nacional de Estadísticas (INE)

En la Figura No. 5 se observa la composición de los cuentapropistas respecto a su edad indicando que para el año 2013 un 19\% están en el rango de 25-35 años, un 22\% de mayores de 35 hasta 45 y el 29\% del etario de mayores de 45 a 60 años, lo que muestra que población entre mayor edad va haciendo más susceptible a ser cuentapropista, como se ha indicado en la investigación muchos de ellos lo realizan por subsistencia o por oficio, asimismo en el año 2015 la composición sigue siendo similar que en años anteriores lo cual denota que en el último trienio no se han formulado políticas públicas respecto a este fenómeno. Se destaca principalmente los mayores de 60 años que deberían estar pensando en jubilarse, sin embargo, debido por la naturaleza del mercado laboral hondureño siguen siendo laborando y representan un 16.3\%; lo que hace reflexionar que estamos haciendo por ellos como país, aunque su producción sea a pequeña escala, son los que ayudan a mantener baja la tasa de desempleo. Y es de resaltar que para el 2015 solo un $0.45 \%$ de las cuentas propias tenían seguridad social, esto desmejora el bienestar de la población presente y futura. No se les está proveyendo de oportunidades para alcanzar una vejez digna. 


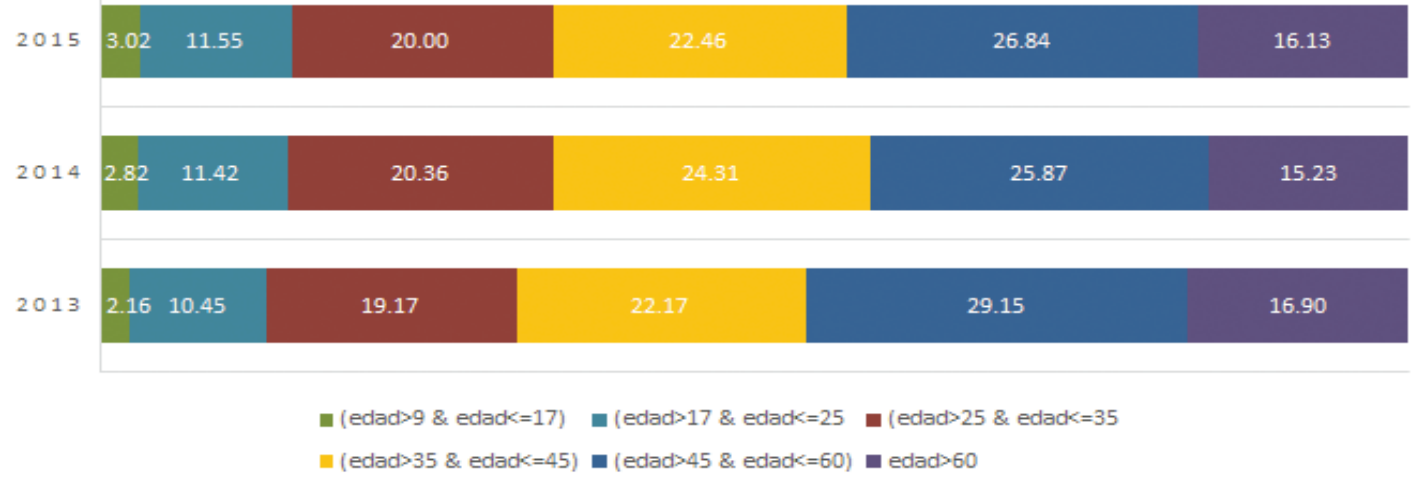

Figura No. 6. Porcentaje de Cuenta Propias por Rango de Edad periodo 2013-2015 Fuente: Elaboración propia con datos del Instituto Nacional de Estadísticas (INE).

Debido a las necesidades del núcleo familiar el jefe de hogar o el generador de los ingresos económicos en cierta manera se ven forzado a insertarse al mercado laboral para cubrir las necesidades de los que dependen de él. En el caso de Honduras se ve reflejado en el porcentaje de casados y de Unión libre que son cuentapropistas para el 2013 representaron un $67.2 \%$ de los ocupados por autoempleo, con una disminución de $2.36 \mathrm{pp}$ para el 2014 y para el 2015 de cada 100 cuentapropistas un 68 indicaron estar casado o en unión libre. Cabe destacar que la población joven también se inserta como empleado autónomo para el 2015 de cada 100 cuentapropistas 25 de ellos eran jóvenes inferiores 2 en relación al 2014 (27 jóvenes). Dejando entre ver la importancia de esta categoría ocupacional como generadora de ingresos y de empleo por la irregularidad del mercado laboral siendo más factible desempeñars

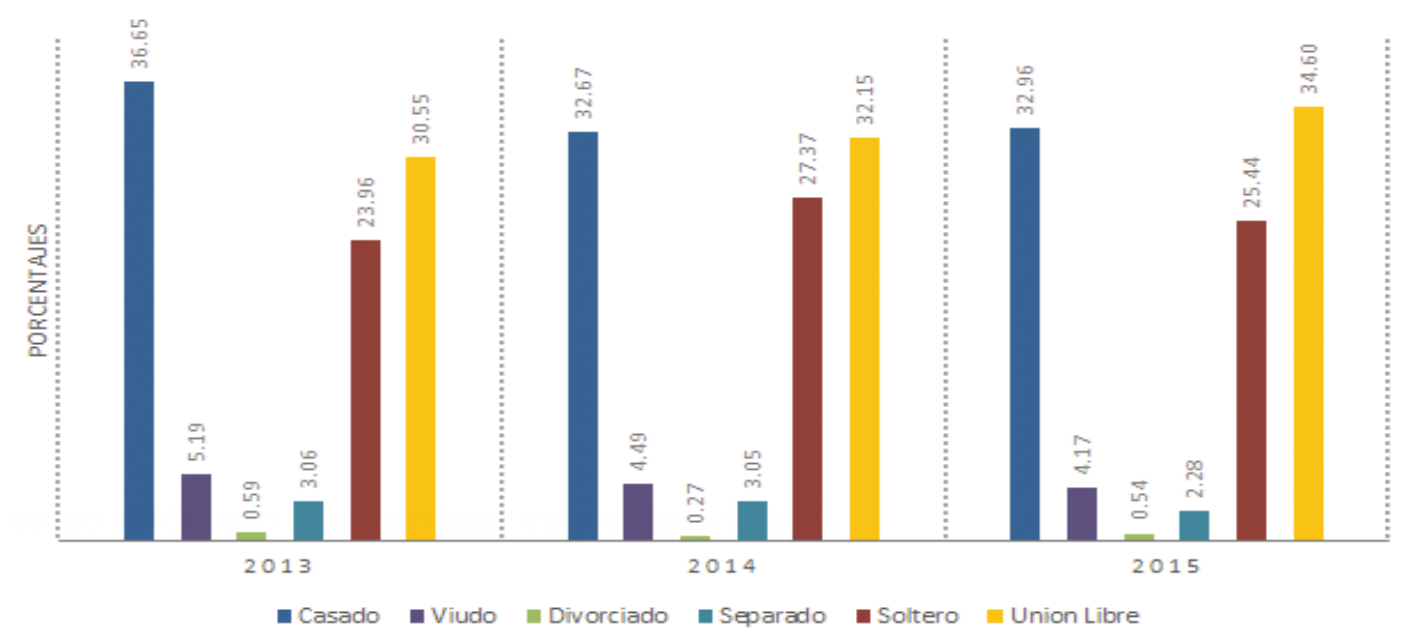

Figura No. 7. Estado Civil de los Cuenta Propia periodo 2013-2015 Fuente: Elaboración propia con datos del Instituto Nacional de Estadísticas (INE). 
Los jefes de hogar no necesariamente es el individuo que aporta mayor ingreso en el hogar sino aquel que los demás miembros lo reconocen como jefe. La figura No. 7 nos muestra los jefes de hogar que indicaron ser cuentapropistas, destacando en el periodo de estudio más del 50\% correspondía a ellos. Como se mostró en la figura No. 6 los individuos con responsabilidades son parte significativa del autoempleo ya que se ven forzados a cubrir las necesidades de su familia.

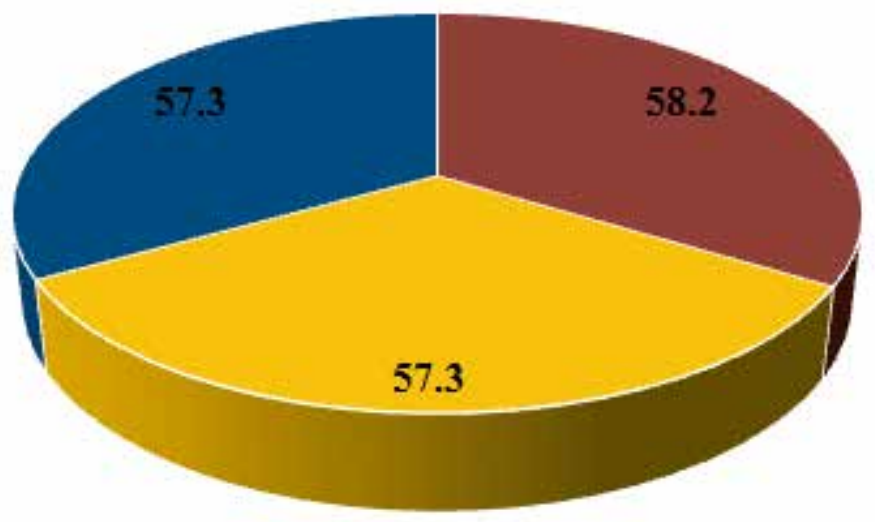

. $2013 \quad$ : 2014 : 2015

Figura No. 8. Porcentaje de Jefes de Hogar que son

Cuentapropistas periodo 2013-2015

Fuente: Elaboración propia con datos del Instituto Nacional de Estadísticas (INE).

En el figura No. 8 como era de esperarse son los jefes de hogar los que tienden a emprender a ser cuenta propias debido a que su responsabilidad es llevar alimentos a sus hogares, en promedio en el último trienio $57 \%$ del total de los cuenta propia son jefes de hogar, sin embargo, es destacable que también el cónyuge se inmiscuya en ser cuenta propia ya que representan $20 \%$ del total de cuanta propias, otro aspecto importante es que los hijos en promedio en los tres años representan 15\% de los cuenta propias, esto significa que en su mayoría los cuenta propia emprenden negocios familiares y en muchos casos los hijos y el cónyuge están involucrados en el negocio de la familia ya sea directa o indirectamente. 


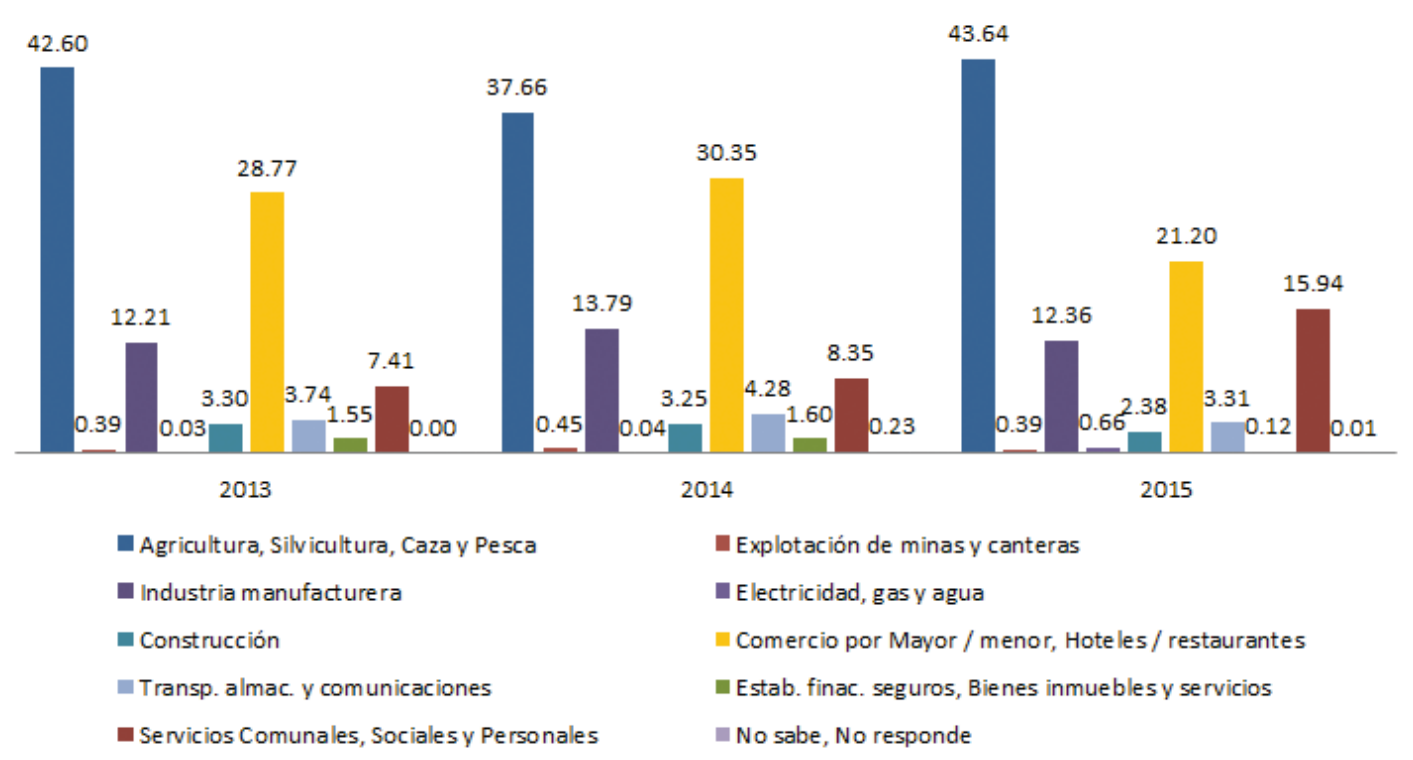

Figura No. 9. Ramas de Actividad de los Cuenta Propias

(Porcentajes, periodo 2013-2015)

Fuente: Elaboración propia con datos del Instituto Nacional de Estadísticas (INE).

El sector agrícola se ha caracterizado por ser el mayor empleador en Honduras, para el año 2013 representó $35.8 \%$ de los ocupados y con aporte al Producto Interno Bruto (PIB) de $13.85 \%$ para el 2014 el tercer sector de importancia en la economía de Honduras, no obstante, tiene el menor de acceso a créditos (5.1\% para el 2014). En caso de Cuenta propias no es la excepción ya que en 2015 representaron $43.6 \%$ de ellos pertenecen al sector agrícola, $21.20 \%$ al sector comercio y un $12.36 \%$ al sector industrial, son sectores que se caracterizan por un bajo nivel de especialización y capital humano mínimo, en caso de comercio se asemeja más a la población que quiere emprender.

Los cuenta propia o autónomos que indicaron tener educación superior mencionaron en la encuesta estar insertados en sector de Comercio por Mayor/Menor, Hoteles / restaurantes (36.67\%), Establecimiento financieros, seguros, Bienes inmuebles y servicios (24.86\%) y Servicios Comunales y sociales y personales un (13.53\%); esto debido a la naturaleza de cada actividad, ya que pueden prestar sus servicios en caso de ser abogados, contadores, entre otros que brindan servicios personalizados, en caso de comercio es más necesario considerar que son aquellos emprendedores se rubro se caracteriza por ello. Los no universitarios tienen a predominar en el sector agrícola (43.9\%); influenciado por el dominio, el nivel educativo, comercio (28.5\%) e industria manufacturera (12.4\%); ya que existen personas que manufacturan en hogares productos y lo venden por motivos estacionales o empresas de manufactura no constituidas. 


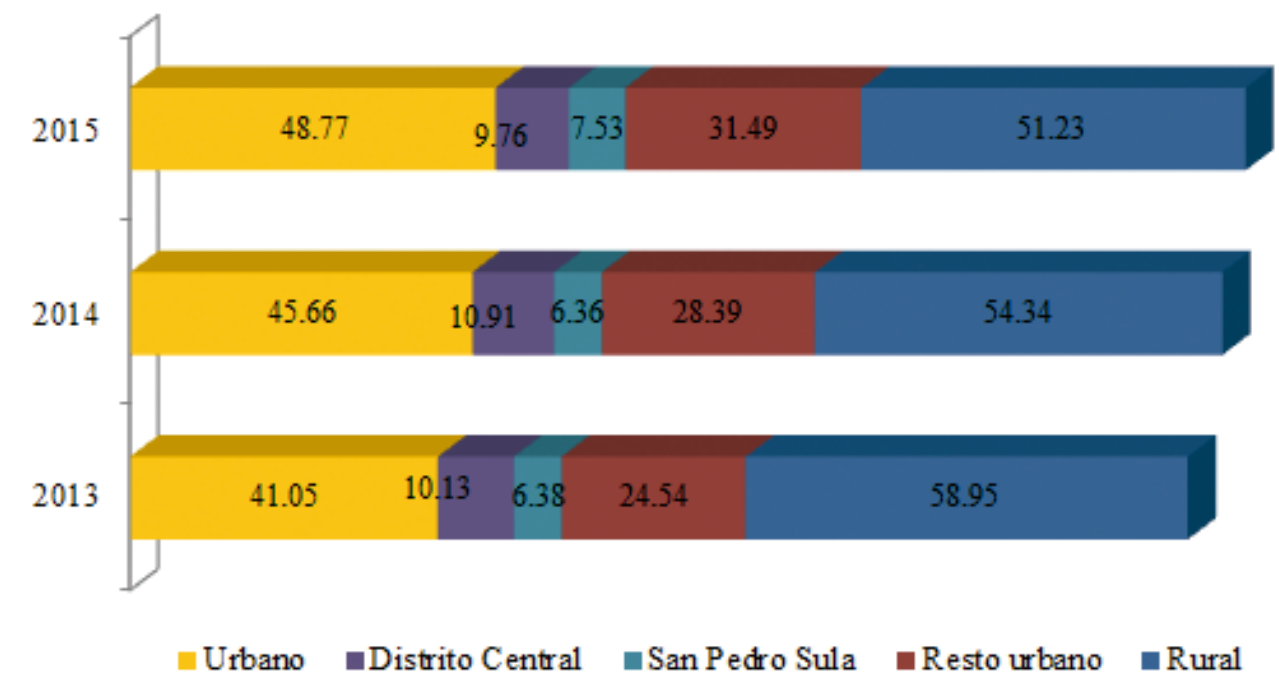

Figura No. 10. Cuentapropistas por Dominio (2013-2015) Fuente: Elaboración propia con datos del Instituto Nacional de Estadísticas (INE).

La importancia de la educación al momento de insertarse en el mercado laboral formal es de relevancia por las exigencias de una mano de obra calificada. Observándose en la figura No. 10 que la población con un nivel educativo bajo (sin nivel y primaria) tienen una significativa participación en el autoempleo dejando la interrogante si son población que lo hacen por subsistencia. En el 2015 un 60.42\% de la cuenta propia contaban con educación primaria superior en $40.7 \mathrm{pp}$ a los que poseían secundaria y solo un $4.57 \%$ tenían educación superior. En relación al total de ocupados de cada 100 empleados 38 contaban con nivel educativo primario correspondiente a la población asalariada y 46 a los empleados por cuenta propia; con educación secundaria 46 de cada 100 son empleados asalariados y 29 a empleados autónomos y a nivel superior 76 son asalariados y 19 de cada 100 autoempleo, indicando que la población con nivel educativo bajo tiene a incursionar mayormente como autónomos prestando su servicios con salarios relativamente bajos y los autónomos con educación superior lo desarrollan como se menciona en el documento por emprendedurismo o se desempeñan como consultores independientes. 


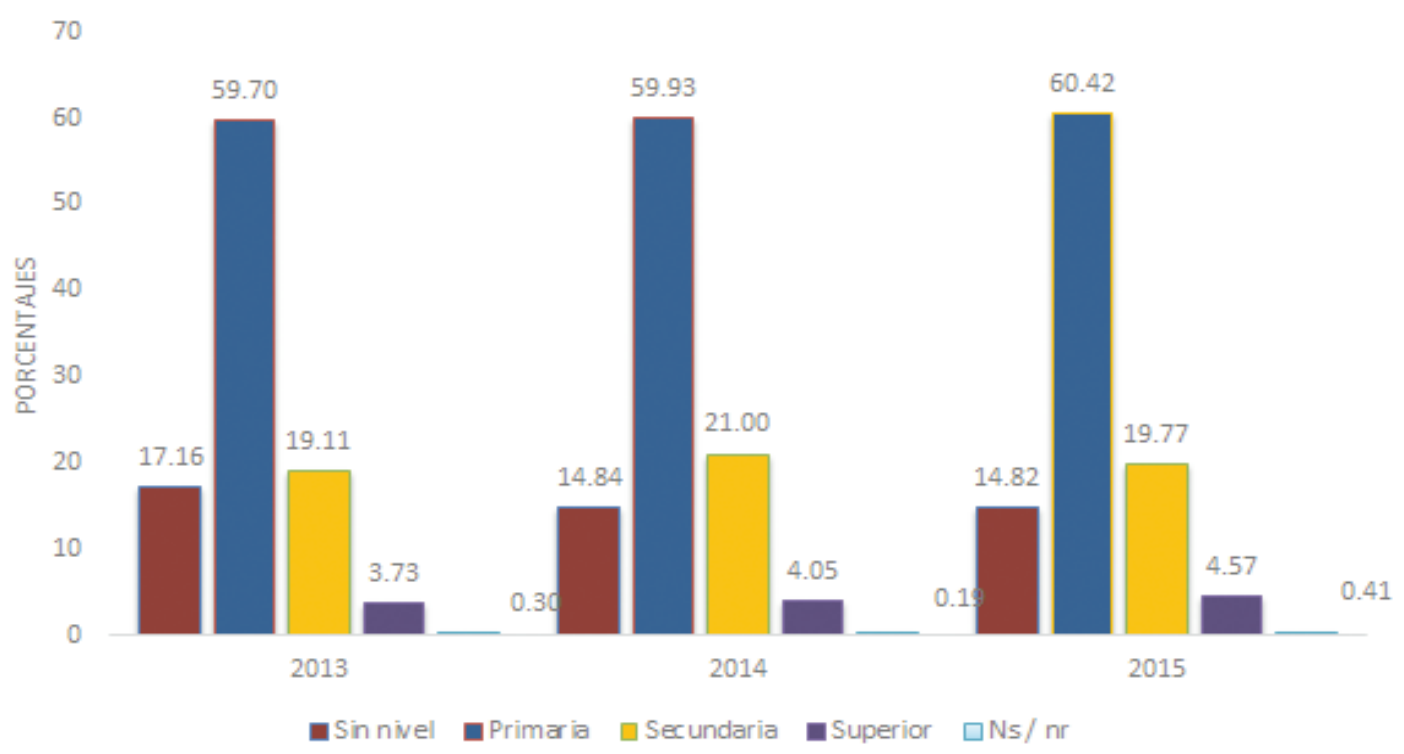

Figura No. 11 Porcentaje de Cuentapropistas por Nivel educativo alcanzado Fuente: Elaboración propia con datos del Instituto Nacional de Estadísticas (INE).

Entre las principales variables de estudio se encuentra el ingreso que perciben las personas que se encuentran ocupadas y que a su vez son remuneradas, por lo cual se divide el total de ocupados en cuatro ramas principales, las cuales son: Empleados Públicos, Empleados del sector Privado, Empleados Domésticos y Cuenta Propias, lo cual dio como resultado los siguientes datos en el Figura $\mathrm{N}^{\circ} 11$.

Es de resaltar que los empleados públicos en el año 2015 obtuvieron en promedio mejores ingresos que los Cuenta Propia, aproximadamente 217\% más, y 121\% más que los empleados públicos, no obstante, los cuenta propia perciben en promedio $31 \%$ mayores ingresos que los empleados domésticos y $43 \%$ menos que los empleados del sector privado, mostrando que pese a que el sector de los cuenta propia es el mayor generador de empleo, sus bajos ingresos pueden estar orientados al nivel educativo alcanzado y al rubro que se orientan sus actividades principalmente la agricultura y con niveles de educación primaria en su mayoría.

\begin{tabular}{|l|l|l|l|l|}
\hline Ingreso & Empleado Publico & Empleado Privado & Empleada Domestica & Cuenta Propia \\
\hline 2013 & 11209.98 & 5388.58 & 2865.71 & 3735.15 \\
\hline 2014 & 12204.60 & 5967.80 & 3016.89 & 3658.62 \\
\hline 2015 & 11928.20 & 5827.34 & 3176.85 & 3757.63 \\
\hline
\end{tabular}

Figura No. 12. Ingreso Promedio de los Cuenta propias (2013-2015) Fuente: Elaboración propia con datos del Instituto Nacional de Estadísticas (INE). 


\section{CONCLUSIONES}

El mercado laboral merece una especial atención no sólo a nivel de los indicadores de desempleo y de las ramas de actividad en las que tiene una dinámica visible, sino también en la estructura del mismo refiriéndonos de manera particular a la composición del sector de los ocupados, para el caso particular de los ocupados que se encuentren en cuenta propias o autoempleo.

Las características de la informalidad que suelen estar presentes en la vida económica de los países en desarrollo con una mayor intensidad, son una plataforma que inciden directamente sobre la formación y persistencia de los cuentapropistas en el mercado laboral. En ese sentido para el caso de la retribución monetaria que perciben los cuenta propia refleja el nivel educativo y la actividad económica en el cual se desarrollan, así como que la mayor parte de ellos se encuentran en la zona rural para el 2015 el 51.3\% en comparación con el 2013 que fue del 58.95\%, esto refleja parte de los movimientos migratorios a nivel interno de la zona rural a la urbana.

Considerando la definición de emprendedor, suele confundirse o tener la noción de que es similar al de cuenta propias, sin embargo, existe una diferencia entre ambos y es la razón por la cual entran a ser ocupados por cuenta propias, es decir si uno entra por la idea de innovar en algún producto o servicio, el otro procura tener un ingreso, sin que estos se traduzcan en ganancias, sino que en ingresos para el sustento de su hogar, el emprendedor busca la ganancia del trabajo independiente que desarrolla.

Las políticas laborales necesitan conocer más a fondo la composición de los ocupados en el país, contar con un análisis que le brinde al tomador de decisiones la información referente para este caso sobre el comportamiento de los cuentapropistas que permitan identificar elementos necesarios sobre su adecuación en el sector informal de la economía con el propósito de desarrollar estrategias en torno a la mejora de las condiciones empresariales en las cuales se desenvuelven este sector de los ocupados. 


\section{REFERENCIAS BIBLIOGRÁFICAS}

Amarante. V, Perrazo I (2013. Trabajo por cuenta propia y monotributo en Uruguay. Recuperado de https://www.colibri.udelar.edu.uy/bitstream/123456789/4226/5/dt-0413.pdf.

Asociación Internacional de Seguridad Social (2012).Manual sobre la extensión cobertura de seguridad social a los trabajadores por cuenta propia. Recuperado de https:// www.google.hn/url? sa $=\mathrm{t} \& \mathrm{rct}=\mathrm{j} \& \mathrm{q}=\& \mathrm{esrc}=\mathrm{s} \&$ source $=$ web $\& \mathrm{~cd}=1 \& \mathrm{cad}=\mathrm{rja} \& u a c t=8 \&-$ ved=0CBwQFjAAahUKEwi-5JD6o_PHAhVDmR4KHT3gAIQ\&url=https\%3A\%2F\%2Fwww.issa.int\%2Fdetails\%253Bjsessionid\%3D5042B4E5AF3EB1B7E1172FCC53F45A1F\%3Fp_p_id\%3DDetailDocumentPortlet_WAR_ISSA_Event_Documentsportlet_INSTANCE_JPFQhjRPI0Fk\%26p_p_lifecycle\%3D2\%26p_p_state\%3Dnormal\%26p_p_mode\%3Dview\%26p_p_cacheability\%3DcacheLevelPage\%26p_p_col_ id\%3Dcolumn-1\%26p_p_col_count\%3D1\%26_DetailDocumentPortlet_WAR_ISSA_ Event_Documentsportlet_INSTANCE_JPFQhjRPI0Fk_uuid\%3D70946175-2f0f-4ca8832c-a4fcc490fe96\%26_DetailDocumentPortlet_WAR_ISSA_Event_Documentsportlet_INSTANCE_JPFQhjRPI0Fk_name\%3D3-Extension-Handbook-Self-employed. pdf\%26_DetailDocumentPortlet_WAR_ISSA_Event_Documentsportlet_INSTANCE_JPFQhjRPI0Fk_mimeType\%3Dapplication\%25252Fpdf\&usg=AFQjCNHbshZVHEmtdrWojH_Jgu8UjOAAkw\&sig2=4LYYnmgkVJ81LrtyxbNqxg

Banco Mundial (2013). Informe sobre el desarrollo social. Recuperado de http://siteresources. worldbank.org/EXTNWDR2013/Resources/8258024-1320950747192/8260293-1322665883147/ Overview_Spanish.pdf

Carrasco, I. y Castaño, M.S. (2008) El emprendedor Schumpeteriano el contexto social. Revista ICE. $\mathrm{N}^{\circ}$ 845. noviembre.diciembre. Universidad de Castillo-La Mancha. http://www.revistasice.com/cachepdf/ICE_845_121-134_F6565B36FDFD765A932BB8BEEE9AAB41.pdf

Cea. S, Contreras.M, Martínez. C,\& Puentes. E, (2009). Trabajadores por cuenta propia: ¿quiénes son? ¿De dónde vienen? ¿Para dónde van?. Recuperado de http://www.previsionsocial.gob.cl/subprev/?wpfb_dl=377

Lepore, E., \& Schleser, D. (2004). LA HETEROGENEIDAD DEL CUENTAPROPISMO EN LA ARGENTINA. Buenos Aires, Argentina. Obtenido de http://www.trabajo.gob. ar/downloads/biblioteca_estadisticas/toe4_06heterogeneidad.pdf

Instituto Nacional de Estadísticas (2013). Encuesta permanentes de Hogares de Propósitos Múltiples

Instituto Nacional de Estadísticas y Censos (2014). Encuesta Continua de Empleo El 
empleo informal en Costa Rica. Recuperado de http://www.inec.go.cr/wwwisis/documentos/INEC/ECE/2014/ECE-Empleo-Informal-CR.pdf

Organización Internacional de trabajo (2006).Panorama laboral de América Latina y el Caribe. Recuperado de http://www.ilo.org/wcmsp5/groups/public/---americas/---rolima/documents/publication/wcms_186248.pdf

Organización de Internacional del Trabajo (2013). La medición de la informalidad: Manual estadístico sobre el sector informal y el empleo informal. Recuperado de http:// www.ilo.org/wcmsp5/groups/public/---dgreports/---dcomm/---publ/documents/publication/wcms_222986.pdf

Organización de Internacional de Trabajo (2014). Panorama laboral Temático. Recuperado de http://www.ilo.org/wcmsp5/groups/public/---americas/---ro-lima/documents/ publication/wcms_315054.pdf

Schumpeter, J. A. (1934). The Theory of Economic Development, Cambridge, MA, Harvard University Press.

Secretaria de coordinación general de gobierno (2014). Ley de protección laboral para los trabajadores autónomos por cuenta propia y no asalariados. Recuperado de http:// transparencia.scgg.gob.hn/descargas/temp_files/circulares/CIRCULAR-CGG-0522014_a_Secretarios_de_Estado_ref_Observaciones_a_la_Ley_de_Proteccion_Laboral_para_Los_Trabajadores_Au.pdf 